\title{
How Far Can Data Loops Go?
}

\author{
JOHN R. PIERCE, FELLOW, IEEE
}

\begin{abstract}
The switching of addressed blocks of data through a network (message switching) is particularly suited to the sort of inquiry-response communication characteristic of many business transactions. In a system of interconnected loops, efficient message switching can be attained with distributed control rather than common control. The initial capital investment in such a system would be low and the investment would grow only as the system grew.
\end{abstract}

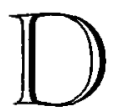

ATA transmission can be local transmission in a building or a complex of buildings; it can be transmission within a geographically extensive but highly integrated complex of machines (an airline reseryation service, for example), or it can be communication among a variety of separately owned machines, as in the ARPA network [1] and in some banking applications. In this last case, the compatibility required for communication is attained partly by agreement among those who desire to communicate with one another and partly by acceptance of certain operating characteristics of the network over which the communication is carried out, as, the switched telephone network or the ARPA network.

Communication among separately owned computers and terminals is bound to grow, especially in such widespread commercial applications as verification of credit cards. Thus, widespread multiuser networks will come into being. These may be either common-carrier networks, or networks operated to serve a consortium of users such as banks (which can operate in a single state only but carry out a variety of transactions with other banks and with various businesses). Such multiuser networks will necessarily involve switching, whether this is explicitly built into their operation or is somehow supplied as a part of the users' hardware and software.

The switching may be line switching. That is, a circuit may be assigned exclusively to a pair of users for the time needed to complete a transaction. Line switching is well suited to lengthy transactions, as, the transmission of a day's accumulated data. Or, switching may be message switching. That is, a block of data addressed by one user to another finds its way through circuits that are not assigned exclusively to a pair of terminals, but on which other addressed blocks of data may be interspersed between those sent between a particular pair of terminals.

Manuscript received September 22, 1971; revised December 30,1971 .

The author is with the Department of Electrical Engineering, California Institute of Technology, Pasadena, Calif. 91109.
Message switching avoids the time wasted in setting up a particular circuit for exclusive use. Message switching is particularly suited to short inquiry-response transactions, such as credit-card verifications and various other financial or information transactions.

Message-switching networks can be organized in what might be thought of as series or parallel configurations. Fig. 1 illustrates a parallel configuration. Customers are connected by individual circuits to nodes, which in turn are interconnected by trunks. Fig. 2 illustrates a series or loop system. In such a system the customers are connected in a local loop or ring. Local loops can be connected to a trunk loop by means of nodes. These nodes can involve buffering, so that the rate on a trunk loop need not be the same as the rate on a local loop that is connected to it.

In the operation of a parallel system such as that of Fig. 1, each customer might simply direct a message at a node and take his chance that his message would not overlap a message from another customer. With such operation, the efficiency of a parallel system would be very low, for traffic would have to be very light in order to avoid serious overlap. Rather, in parallel systems it seems essential that the nodes provide a fairly sophisticated form of common control; that they interrogate customers concerning demand for service according to a plan, provide service to customers, and assign messages to trunks in a way that assures both small delay and efficient use of trunks.

A series or loop system such as that shown in Fig. 2 can operate in a variety of ways. In the Collins C-8500 direct digital control system a separate time slot is allocated to each multiplex channel unit. ${ }^{1}$ This is simple but it would be unsatisfactory in long-haul communication because of the inefficient use of the circuit.

At least one system has used a geographically extensive loop [2]. In this loop, all messages go to or from a central processor, so it does not exemplify a multiuser network.

IBM has used the serial or loop configuration for transmission within a restricted geographical area [3], [4]. This system allows a more efficient use of the channel by allowing an "area station" to write into any blank frame which is generated by a system controller.

All the loop systems previously referred to interface with other nonloop transmișsion systems by means of complex common control devices. Thus, none is the sort of interconnection among loops that is shown in Fig. 2.

\footnotetext{
1 See company brochures.
} 


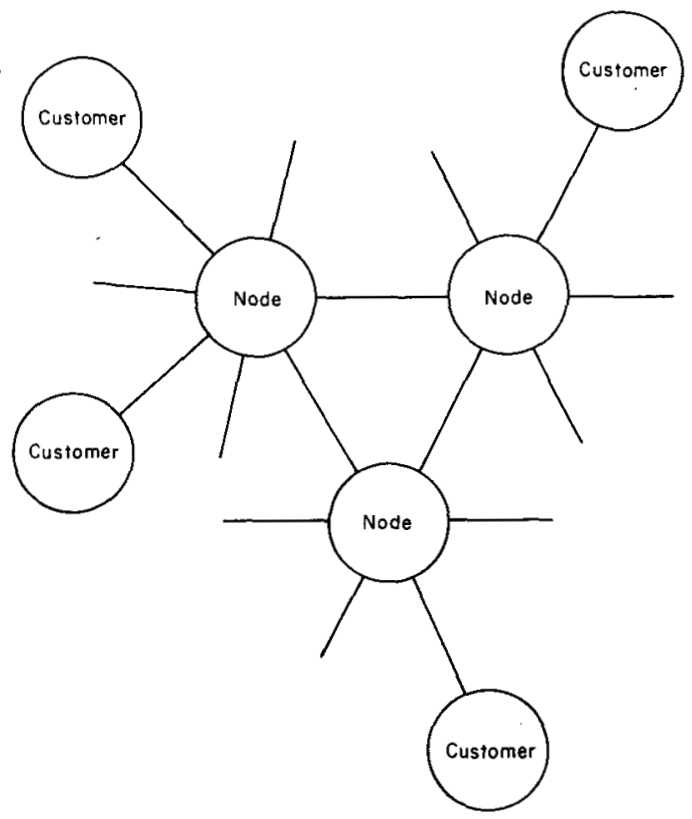

Fig. 1. Parallel system.

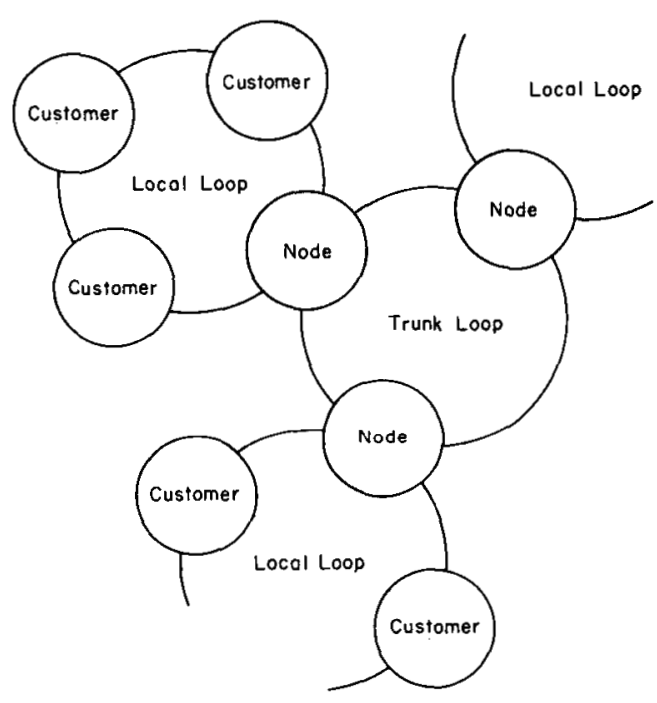

Fig. 2. Series (loop) system.

Another experimental loop system also made use of common control [5].

Pierce et al. [6] have shown that a loop system can be so designed that the nodes can be very simple and merely provide clock for the loop, generate formatted message blocks, provide buffering, determine whether a message address is the same as a wired-in address, and perform a few simple wired-in logical functions.

Efficient operation is attained, not by sophistication at the nodes, but because a customer must wait for access until a message block that is marked empty passes his terminal. In transferring a message to a trunk loop a node must hold a message block in buffer until a blocked marked as empty passes the buffer on the trunk loop. Analyses show that the average waiting time is modérate [7], [8].
By using this technique, a very extensive system such as that indicated in Fig. 3 could be built up. The $A$ boxes in the figure provide clock, generate empty data blocks, and perform simple supervisory functions. The $B$ boxes are customer terminals. Regional and national loops are interconnected by means of $C$ boxes. Addresses would be made up of a number on the local loop, a number of the local loop, and a number of the regional loop. The $C$ boxes that interconnect loops are not common control devices. In transferring messages from one loop to another they merely compare a part of the address of a block (or frame) with a wired-in address.

Thus, a loop switching system can differ from other line or message switching systems in one important way. An efficient loop systems can be designed without any sophisticated common control. The cost of switching is distributed among the customers' terminals and equipment at the nodes.

Such a system can be designed for a large ultimate capacity by establishing at the start an adequate numbering plan, by ascertaining the nature and availability of common-carrier circuits, and by planning an ultimate topology of the network. However, the system could be brought into being as service is needed. In a local loop, presumably two circuits would run from a wire center to each customer and would be interconnected within or among wire centers to form the loop, somewhat as illustrated in Fig. 4. In this way it would be very easy to add customers to a loop without altering the circuits used for existing customers. Further, simple equipment could monitor the output of each incoming circuit and bridge across the circuit to and from a customer if it ceased to operate.

In Fig. 4, two wire centers are involved in a single local loop; one houses the $A$ box. The loop serves a number of customers, one of whom has two $B$ boxes. The $C$ box interconnecting the local loop with a regional loop would be located in one or the other of the wide centers. Similarly, regional and national loops would be made up of existing circuits between existing wire centers, and $B$ and $C$ boxes would be located at wire centers. Switching cost, and transmission cost, for that matter, would grow roughly in proportion to the number of customers. Because the customer's terminals and the equipment at the nodes are both simple, the capital investment required for start-up would be small, and the investment would grow slowly as customers were added and as lowspeed trunks were replaced with high-speed trunks.

In contrast, in order to offer widespread service with a line-switching or message-switching system, which requires common control, a considerable capital investment must be made initially before service can be offered at all. This could prove financially embarrassing if the demand for and growth of service proved to be small.

Loop systems have another advantage over other message-switched and over line-switched systems. A large organization may require service at several points within 


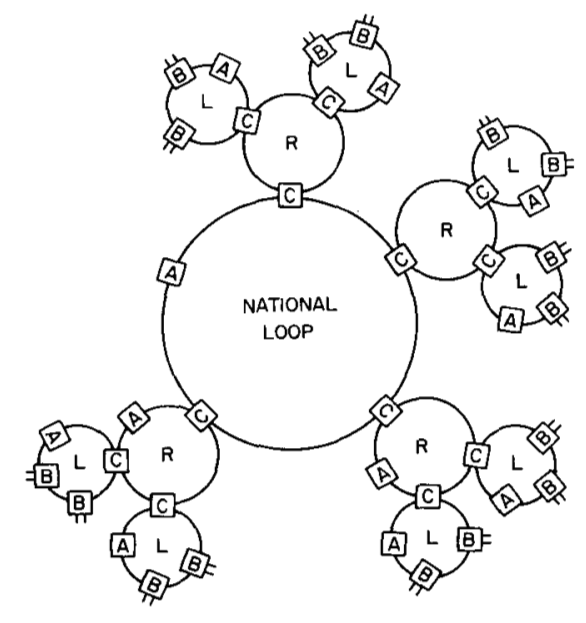

Fig. 3. Loop system with local, regional, and national loops.

one building. If service is provided by means of a loop system with a high bit rate, one circuit to and an another circuit from the premises are sufficient; users on the premises can be connected in series to form part of the loop. Other systems would require as many pairs of circuits as there are users. Although these circuits could conceivably be of lower speed than the loop circuits, it seems likely that the total cost would be greater.

The advantages cited are of a general nature, but digital communication systems must thrive in a real world and their performance must be related to the nature of real data traffic.

If customers transmitted data continuously over a fixed route, private line service, synchronous or asynchronous, would clearly be optimal, and any consideration of switched data systems would be academic.

If data messages were long compared with the setup time for line switching $(2-10 \mathrm{~s}$ for present telephone switching; perhaps a tenth of this is feasible), line switching would be suitable and economical for customers who needed intermittent service.

It appears, however, that much future data traffic will be of an intermittent inquiry-response nature and that many messages may be quite short. This would certainly be true for credit-card verification and for many messages involving transfer of funds, ordering goods, or information retrieval. Private lines seem ill suited to such traffic, and line switching is ill suited if the data rate is so high that message length is very short compared with setup time.

Message switching seems ideally suited for inquiryresponse traffic; loop systems appear to allow the initiation and growth of message switching with a small capital outlay.

However, it would be wrong to assume that message switching and loop systems would be advantageous for short messages only. It is possible that some customers who use private lines do not transmit continously, but rather, use private lines so as to have a circuit instantly available. A high-capacity loop system with addresses

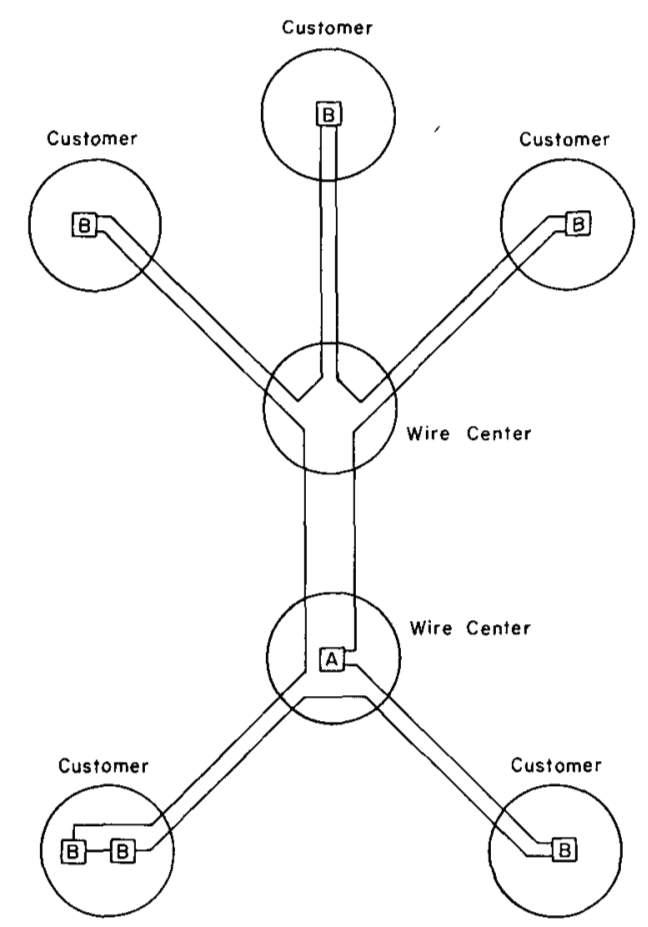

Fig. 4. Circuit paths in a local loop involving two wire centers and several customers.

wired in at the customer's terminals could provide almost instantaneous transmission. If it was engineered so as not to overload, it would give a customer essentially the same service as a private line. If the customer used the service for a reasonable small fraction of the time, the loop system could be as satisfactory as and cheaper than a private line.

Several general objections have been raised to loop systems.

1) Reliability. A break in a loop denies service to all customers on the loop.

2) Privacy. One customer's messages pass through another customer's premises.

It seems to the writer that a satisfactory degree of privacy and reliability can be attained. As to reliability, simple circuits can automatically bridge across an inactive $B$ box. If the topology is as shown in Fig. 4, simple circuits at a wire center can bridge defective portions of a loop. The error rate of $T_{1}$ circuits is presumably very low. The reasonable approach would seem to be to attempt to attain adequate privacy and reliability rather than to forgo possible advantages of loop systems on the basis of reliability or privacy.

There are what seem to the writer to be more compelling practical objections to the loop system.

3) The cheapest and most widely available digital transmission system suitable for local loops is the $T_{1}$ carrier whose 1.5-Mbit rate is uncomfortably high even for computers.

4) The $T_{1}$ carrier is widely available but it is not available everywhere and requires repeaters every mile. It may be too expensive to install. 
5) The customers' terminals in a loop system, though fairly simple, may be too expensive for very small users; i.e., for the verification of credit cards in a small store.

6) When traffic becomes very large, some more complicated systems might be more economical.

The objection to $T_{1}[3$ ) and 4) above] may not be as serious as it seems. Buffering at terminals could take care of the speed problem. For customers within a mile of a central office, no $T_{1}$ repeaters would be needed between the customer's premises and the central office. Further, data transmission needs might ultimately bring into being a digital transmission system somewhat slower than $T_{1}$, which could tolerate less perfect cables and larger repeater spacings.

Objection 5) might be overcome by means of a simple multiplexer in a central office, which would enable one loop customer terminal at the central office to serve a number of small slow intermittant users over low-speed unrepeatered lines.

In regard to objection 6), it may be that the most immediate problem of economics is that of getting started, not that of providing service many years from now.

In conclusion, loop switching systems offer several possible advantages.

1) Low initial capital investment, the investment growing with the number of customers.

2) One high-speed data circuit to a location and one high-speed data circuit from the location can serve many independent users at the location.

3) If addresses are wired in at the customer's equipment, the service becomes equivalent to a private line, that is, instantly available, but the cost will be lower if the customer's traffic is sufficiently intermittent.

The question concerning loop switching systems is not whether or not they can be built and used; that has been demonstrated. The questions are rather: can a widespread multiuser system be built economically? Are cer- tain objections as serious as some maintain, and if they are, can they be overcome? If loop switching systems are sound will someone have the enterprise to offer a widespread multiuser service?

\section{REFERENCES}

[1] I. Roberts. "A computer network designed to achieve resource sharing," in 1970 Spring Joint Computer Conf., AFIPS Conf. Proc., vol. 36. Montvale, N. J.: AFIPS Press, 1970.

[2] H. K. M. Grosser and F. J. Schramel, "Data transmission and switching equipment for the seat reservation system of United Airlines (1)," Philips Telecommun. Rev., vol. 24, pp. 13-24, Feb. 1963.

[3] J. Martin, Teleprocessing Network Organization. Englewood Cliffs, N. J.: Prentice Hall, 1970, pp. 122-125.

[4] E. H. Steward, "A loop transmission system" in Proc. 1970 Int. Conf. Communications, San Francisco, Calif., June 8-10.

[5] W. D. Farmer and E. E. Newhall, "An experimental distributed switching system to handle high-speed aperiodic computer traffic," in Proc. HCM Symp. Problems on the Optimization of Data Communication Systems, Oct. 13-16, 1969

[6] J. R. Pierce, C. H. Coker, and W. J. Kropfl, "An experiment in addressed block data transmission around a loop," in IEEE Int. Conv. Rec., Mar., 1971, pp. 222-223.

[7] J. F. Hayes and D. N. Sherman, "Traffic analysis of a ring switched data transmission system," Bell Syst. Tech. J., vol. 50, pp. 2947-2978. Nov. 1971 .

[8] B. Avi-Itzhak, "Heavy traffic characteristics of a circular data network," Bell Syst. Tech. J., vol. 50, pp. 2521-2549, Oct. 1971.

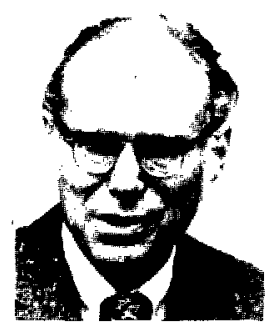

John R. Pierce (S'35-A'38-SM'46-F'48) received the B.S., M.S., and Ph.D. degrees from the California Institute of Technology, Pasadena, in 1933, 1934, and 1936, respectively.

From 1936 to 1971 he was employed by the Bell Telephone Laboratories, Inc., where he became Executive Director of Research, Communication Sciences Division. He has worked in the fields of microwave tubes and communication, communication satellites, and acoustics. He is now Professor of Engineering at the California Institute of Technology.

Dr. Pierce has received various awards including the Morris Liebman Memorial Award, the Edison Medal, the Poulsen Medal, and the Cedegren Medal. He has received seven honorary degrees. 